\title{
A qualitative study of the reconnection between the Earth's magnetic field and an interplanetary field of arbitrary orientation
}

\author{
S. W. H. Cowley \\ Cooperative Institute for Research in Environmental Sciences, \\ University of Colorado and National Oceanic and Atmospheric Administration, \\ Boulder, Colorado 80302
}

(Received July 23, 1973.)

\begin{abstract}
To the present date only the reconnection process for exactly antiparallel fields has been discussed in detail. In magnetospheric terms this restricts us to the consideration only of southward interplanetary fields. The qualitative study presented here shows how reconnection takes place between arbitrarily oriented fields in infinite and finite geometries. The process may best be thought of as a continuous exchange of field-line partners during the time a field line maps into the diffusion region. In a finite geometry the diffusion regions lie on field lines which connect neutral points of the field configuration, and along which a potential drop is imposed. Although the discussion here centers principally on the magnetosphere, the described reconnection process and field topology should also be applicable to other astrophysical problems.
\end{abstract}

\section{INTRODUCTION}

Reconnection between the Earth's magnetic field and the interplanetary field is thought to be the basic driving mechanism of plasma convection within the magnetosphere [Dungey, 1961]. However, only the special case of reconnection between exactly antiparallel fields has to date been discussed, i.e., reconnection between the Earth's field and an interplanetary field directed exactly southward. The purpose of this paper is to obtain a qualitative understanding of the reconnection process which occurs between nonantiparallel fields and to determine how this can be applied to the magnetosphere. This objective is achieved by a two-fold approach. First, the reconnection of nonantiparallel magnetic fields is considered in a system which is infinite along the $X$ line, by a simple modification of the solutions of $Y e h$ and Axford [1970] and Sonnerup [1970]. However, the qualitative results we obtain should not be dependent on the particular solution we choose to consider. Second, the expected topology of the open magnetosphere in the general case is discussed, following Dungey [1963]. We repeat this discussion here in the interests of obtaining a self-contained paper. Our method, as in Dungey [1963], is to

Copyright (C) 1973 by the American Geophysical Union. superpose linearly a uniform magnetic field of arbitrary orientation with a dipole field.

We then combine these two approaches to obtain a consistent qualitative picture of the general reconnection process for arbitrary orientations of the interplanetary magnetic field and the general topology of the magnetosphere. Several authors have recently considered this process briefly, but do not seem to have been led to the picture presented here [Stern, 1972; Gonzales and Mozer, 1973].

Even though the discussion here will center specifically on the magnetosphere, it should also be applicable with suitable modification to other systems of astrophysical interest.

\section{RECONNECTION OF NONANTIPARALLEL MAGNETIC FIELDS IN AN INFINITE SYSTEM}

No complete solution of the magnetic field structure and plasma flow in the vicinity of an $X$ neutral line has yet been accomplished. Sonnerup [1970] and Yeh and Axford [1970] have considered the problem within the framework of collision-dominated fluid theory (the plasma conductivity $\sigma$ being taken to be a scalar) and have considered separately two regimes within the total solution. These two regions are the convection region well away from the neutral line, and the diffusion region immediately surround- 
ing the neutral line. In the convection region the plasma may be considered to be infinitely conducting, so that Ohm's law becomes

$$
\mathbf{E}=-\mathbf{v} \times \mathbf{B} / c
$$

and the field lines are tied into and convect with the plasma. This approximation breaks down in the diffusion region ( $\mathrm{v} \times \mathrm{B} \rightarrow \mathbf{0}$ at the neutral line) and finite conductivity effects become important. In this region, then, the field lines are no longer tied to the plasma and can become broken and reconnected. However, no detailed matching between these two regions has been attempted.

Within the framework of collision-free plasmas there as yet exist no self-consistent solutions of the field and flow near an $X$ neutral line; only neutral sheets and other simple geometries have been considered [Harris, 1962; Alfvén, 1968; Eastwood, 1972; Cowley, 1973]. We therefore consider here the extension of the fluid solutions of Sonnerup and Yeh and Axford for an $X$ neutral geometry to the case where the field lines flowing towards the $X$ line are no longer antiparallel.

In these models the neutral line is considered to be of infinite extent (i.e., $\partial / \partial l \equiv 0$ along the direction of the line), and the field and flow lines are constrained to lie in planes perpendicular to the neutral line. The plasma is assumed to be incompressible ( $\operatorname{div} \mathbf{v}=0$ ). The electric field is parallel to the neutral line and uniform (curl $\mathbf{E}=0$ ) satisfying the previous equation in the convection region and

$$
\mathbf{E}=\mathbf{j} / \sigma
$$

near the neutral line in the diffusion region. In the convection region it is found that the flow lines must traverse two shocks in passing through the system. These shocks thus break up each quadrant of the flow into three regimes: inflow, outflow, and a region between the shocks. In the diffusion region the flow lines are retangular hyperbolae near the neutral line, while the field lines are also systems of hyperbolae. The solutions of Sonnerup for the convection region are a special case of those of Yeh and Axford in the limit of zero radial pressure gradient. In this limit the field lines and plasma parameters between the shocks are uniform and the system is amenable to reasonably simple analytic calculation.

We now consider the addition of a uniform magnetic field $\mathbf{B}_{\|}$to these solutions, the field direction being parallel to the neutral line. This does not affect the dynamic equilibrium of the system since the currents are unchanged and $\mathbf{j} \times \mathbf{B}_{\|}=\mathbf{0}$. However, we must also introduce a new electric field component which lies in the plane of the flow lines given by

$$
\mathbf{E}^{\prime}=-\nabla \times \mathbf{B}_{\|} / c
$$

where $\mathbf{v}$ is the fluid velocity of the new (and old) systems. This field is required by Ohm's law, and is the field required to maintain $\mathbf{E} \cdot \mathbf{B}=\mathbf{0}$. The condition that this field has zero curl everywhere is simply $\operatorname{div} \mathbf{v}=\mathbf{0}$. Thus within the framework of incompressible plasma flow we may simply add a uniform magnetic field and its corresponding electric field to any solution for reconnection of antiparallel magnetic fields and hence obtain a solution for reconnection of nonantiparallel fields. Even in this case more complicated solutions are possible in which the magnetic field parallel to the neutral line is changed in strength across the shocks of the convection region leading to changes in plasma flows parallel to the $X$ line. These will not be discussed, the simplest possible model being sufficient to obtain a qualitative understanding of the processes involved which is all we require here.

The addition of a uniform magnetic field parallel to the $X$ line to the field in the convection region simply twists the field in opposite directions on either side of the diffusion region in an obvious manner. The projection of the field lines on planes perpendicular to the $X$ line remains unchanged. The latter statement is also true for the field lines in the diffusion region. However, within the diffusion region the perpendicular field components become progressively smaller as the $X$ line is approached while the parallel field remains of constant strength. The field line twisting in the diffusion region is therefore more pronounced than in the convection region.

Figure 1 shows the field line structure near the $X$ line, the field lines being projected onto the plane perpendicular to the $X$ line. The perpendicular field components near the $X$ line depend linearly on distance from the $X$ line, i.e.,

$$
B_{\imath}=\alpha_{1}, x_{i}
$$

where $\alpha_{i}=\partial B_{1} /\left.\partial x_{y}\right|_{x=0}$ since the field may be Taylor-expanded about the $X$ point, and since the current there is nonzero.

The field lines are thus systems of hyperbolae. The field lines which map to the $X$ point in this projection will be referred to as the separatrices between the inflow and outflow regions of the system. 


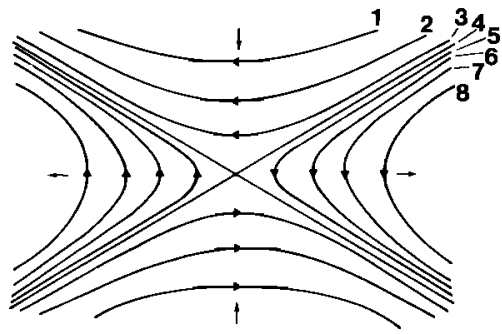

Fig. 1. The field line structure near the $X$ line in the diffusion region: the projection onto a plane perpendicular to the $X$ line.

They also define the boundary surfaces between those field lines which are flowing into the system and those that have been reconnected, in the outflow. Considering the projection of Figure 1, pairs of field lines move toward the separatrices from either side, become broken and reconnected as they map into the $X$ point (i.e., when in the separatrices) and then move away from the $X$ point in the outflow, as in the conventional reconnection picture for antiparallel fields. However, the field lines as they appear if we look down upon this system (i.e., in plan projection) are shown in Figure 2. The field lines of the inflow region marked 1, 2, and 3 are shown by the solid lines, and are sinh-curves. As indicated above, as we progress closer to the $X$ line these field lines become progressively more extended along its direction. The field line which runs down the center of the $X$ is marked $X-X$ in Figure 2. The limiting case of this extension along the $X$ line occurs for the field lines in the separatrices. These lines extend along the $X$ line out to infinity (exponential curves), asymptotically approaching the field line which runs down the center of the $X$, and are shown by the dot-dash line in Figure 2, marked 4. The lines approach the $X$ line in opposite directions on the two leaves of the separatrices shown. Moving through the separatrices and into the outflow region the field-line plan projections are shown as dotted lines marked 5, 6, 7, and 8 in the diagram (cosh curves). The field lines have become reconnected, and their extension along the $X$ line diminishes as we move away from the origin since the perpendicular field strength increases.

This field line structure in the diffusion region leads to interesting consequences in the description of the reconnection process for nonantiparallel magnetic fields. In Figure 3 we have drawn a particular

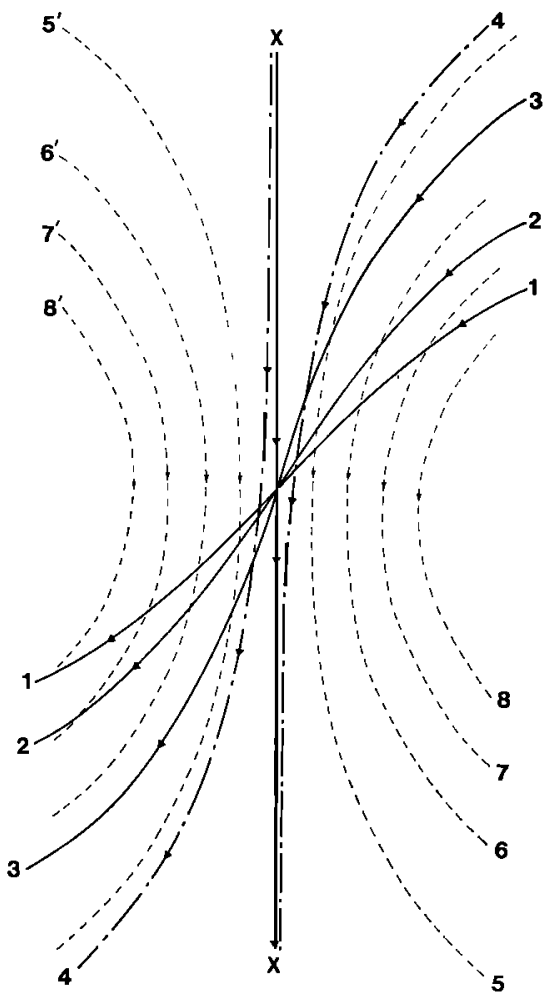

Fig. 2. The field line structure near the $X$ line in the diffusion region: the plan projection.

convection region solution (a Sonnerup solution) surrounding a diffusion region indicated schematically by the octagonal box surrounding the $X$-point. The dotted lines indicate the shock waves. In Figure 4 we show a plan projection of the field lines. We consider the motion of a particular field line, marked

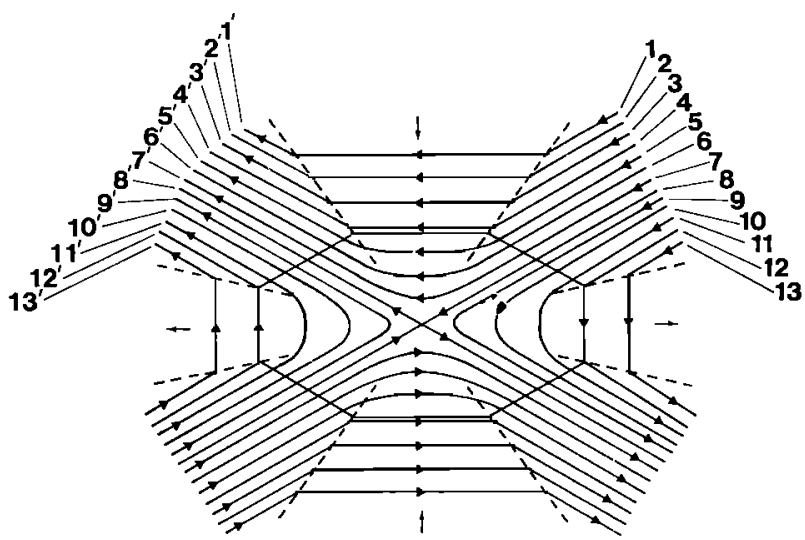

Fig. 3. Reconnection of nonantiparallel field lines: The projection onto a perpendicular to the $X$ line. 


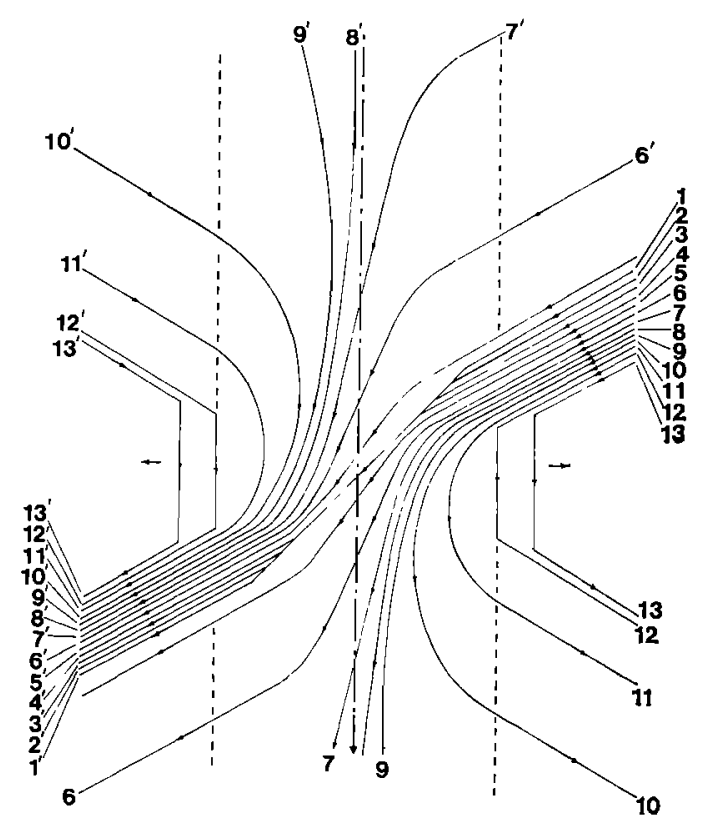

Fig. 4. Reconnection of nonantiparallel field lines: the plan projection.

1-1 on the figures, as it moves through the system. In the convection region we may follow the motion of the field line as it convects, frozen into the fluid. However, since the frozen-in theorem does not apply in the diffusion region we must use our knowledge of its field-line structure to discover where the field line maps once its central portion enters this region. We may, however, continue to follow the ends "of the field line which remain in the convection region. We have chosen the uniform field strength parallel to the $X$ line to be equal to the perpendicular field strength of the inflow region, so that the central portion of the field line 1-1' in plan projection lies at $45^{\circ}$ to the $X$ line (marked by the dot-dash line in plan), and fields of the inflow region on opposite sides of the diffusion region are perpendicular. The line at position 1-1' has two kinks in it as it crosses the shocks on either side of the diffusion region, after which it becomes more nearly perpendicular to the $X$ line since the perpendicular field strength between the shocks is stronger than that of the inflow region. As the line moves down toward the diffusion region (positions $1-1^{\prime}$ to $4-4^{\prime}$ ) the central portion of the field line (in the inflow region) simply appears to become shorter in plan projection as it moves vertically down toward the diffusion region, while the parts of the field line beyond the shocks move slowly sideways since the fluid flow between the shocks has a component in the direction of the outflow. Since the line has remained within the convection region during this period it has simply flowed toward the diffusion region retaining its identity.

The central portion of the field line now enters the diffusion region, so we must now appeal to our knowledge of the field-line structure there. According to the above discussion the field lines become extended along the $X$ line as the move toward it, the extension increasing as we move closer. The extensions are the same for the two ends of the original field line, but in opposite directions, as shown for the lines 5 and $5^{\prime}$. Therefore the original field line does not retain its identity as its central portion moves into the diffusion region but becomes split, and now each convecting end maps through the diffusion region to new convecting partners on the opposite sides of the diffusion region. As they move deeper into the diffusion region the splitting increases (lines $6,6^{\prime}, 7,7^{\prime}$ ) as the extensions along the $X$ line increase until the field-line ends lie in the separatrices. They each now map out to infinity along the $X$ line $\left(8,8^{\prime}\right)$, but in opposite directions. Moving through the separatrices into the outflow of the diffusion region the field lines have become reconnected, but still extend considerably along the $X$ line to partners in the convection region $\left(9,9^{\prime}\right.$, $\left.10,10^{\prime}\right)$. As they move out of the diffusion region their extension along the $X$ line diminishes and they continuously map into closer convection region field lines $\left(10,10^{\prime}, 11,11^{\prime}\right)$. On reaching the edge of the diffusion region this exchange of convection region partners ceases and the reconnected field lines move into the convection region outflow once again retaining their identity $\left(12,12^{\prime}, 13,13^{\prime}\right)$. The fieldline ends to which it is ultimately connected are, of course, those of the field line which was initially placed symmetrically in the other inflow region to the one we have been considering, as indicated in Figure 5, although each piece of the field lines remaining in the convection region throughout the motion has had to map out to infinity and back before this is accomplished.

In order to apply these qualitative results to the magnetosphere we must first consider how this system must be modified in order that field lines mapping out to infinity may be incorporated into a finite geometry. This requires a study of the general topology to be expected for an open magnetosphere and is the subject of the next section. 


\section{GENERAL TOPOLOGY OF THE OPEN MAGNETOSPHERE}

The expected topology of the open magnetosphere in the general case is here investigated by linearly adding a uniform magnetic field of arbitrary orientation to a dipole field. This procedure and its results have previously been described by Dungey [1963] but will be repeated here in the interests of selfcontainment.

The superposition of two fields in this manner is valid only for media with zero conductivity, so that the resultant magnetic fields we produce are not expected to be valid magnetospheric models. However, we expect the field to be topologically correct, particularly if we can show them to be consistent with the reconnection picture discussed above. Such proves to be the case.

The conventional picture of the reconnection of the Earth's field with a purely southward interplanetary field may be represented within this framework by the addition of a uniform field parallel to the dipole moment vector as sketched in Figure 6. An $X$ neutral ring is generated in the equatorial plane at a certain radial distance which corresponds to the dayside and nightside $X$-type neutral lines. The field lines which map from this neutral ring are the separatrix field lines of the $X$ configuration and form the boundary surfaces between the closed, open, and interplanetary field lines. Surface $A$ is the interface between closed and open lines generating in three dimensions a doughnut-shaped boundary, while surface $B$ forms the interface between open and interplanetary lines and generates two cylindrical regions connected to the north and south polar caps (the tail lobes).

This situation is rather a special case; for any other orientation of the uniform field a neutral ring is not generated, rather two neutral points are produced. These points lie in the plane containing the imposed magnetic field direction and the dipole magnetic moment vector. Only in this plane does the imposed field have no azimuthal component; the dipole field has no azimuthal component in all planes containing the magnetic moment vector. In order to discuss the three-dimensional topology we must therefore consider the magnetic field configuration near a neutral point. Close to the null the field may be expanded in a Taylor series so, to lowest order, we can write a matrix expression for the field components of the form

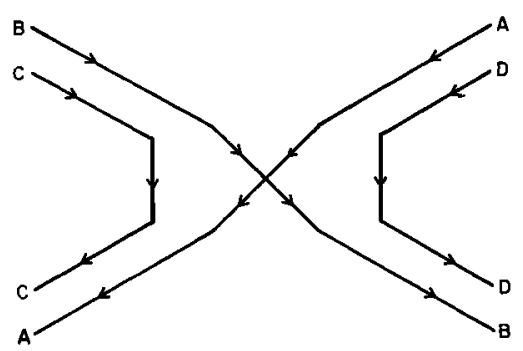

Fig. 5. Showing in plan projection the two inflow convection region field line $A, B$ on opposite sides of the diffusion region which are ultimately connected together, and the resultant reconnected field lines, $C$ and $D$.

$$
B_{\imath}=b_{i}, x_{i}
$$

where $b_{\imath_{1}}=\partial B_{\imath} /\left.\partial x_{1}\right|_{\mathbf{x}=0}$. If there are no currents flowing at the null we have $b_{i j}=b_{n}$ so that the matrix $\{b\}$ is symmetric. It may thus be diagonalized by a suitable rotation of the coordinate system so that

$$
B_{z}^{\prime}=b_{i \imath}{ }^{\prime} x_{i}{ }^{\prime}
$$

However since $\operatorname{div} \mathbf{B}=0$ we have $\operatorname{Tr}\{b\}^{\prime}=0$, and so of the three coefficients $b_{u}$ two will be of the same sign while the third will be of the opposite sign. Dropping primes, let

$$
B_{x}=\alpha x \quad B_{y}=\beta y \quad B_{z}=-(\alpha+\beta) z
$$

where $\alpha$ and $\beta$ are of the same sign. The field lines in the $x, z$ plane are given by

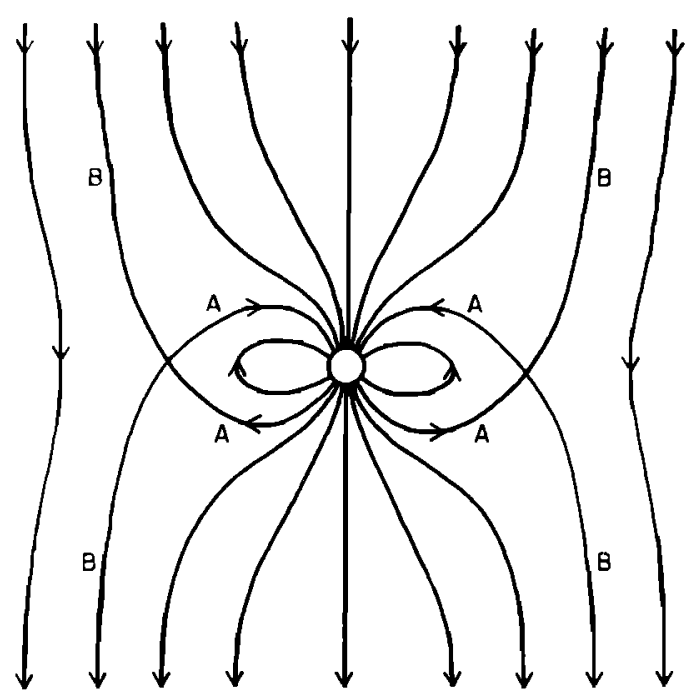

Fig. 6. Field configuration for the special case of a uniform southward magnetic field added to a dipole. 


$$
d x / d z=B_{x} / B_{z}=-[\alpha /(\alpha+\beta)] x / z=-Y_{1} x / z
$$

where $0 \leq Y_{1} \leq 1$. Integrating we obtain

$$
x z^{Y_{1}}=C_{1}
$$

where $C_{1}$ is an arbitrary constant. Similarly, the field lines of the $y, z$ plane are given by

$$
y z^{Y_{2}}=C_{2}
$$

where $Y_{2}=\beta /(\alpha+\beta)$ and $0 \leq Y_{1} \leq 1$.

Therefore the field lines in both these planes will have the form of those shown on the left-hand side of Figure 7. However, the field lines in the $x, y$ plane are given by

$$
x=C_{3} y^{Y_{\mathrm{a}}}
$$

where $Y_{3}=\alpha / \beta>0$. These field lines map into the neutral point and define a surface about it. The field points toward the neutral point if $\alpha, \beta<0$ (type $\mathrm{A}$ in Figure 7), or away from the null if $\alpha, \beta$ $>0$ (type B). Of the field lines above (or below) the surface only one maps into the neutral point and is locally perpendicular to the surface. The field direction along these single lines with respect to the neutral point is in the opposite direction to those in the surface, as shown on the right-hand side of Figure 7. The lines mapping into a neutral point therefore consist of a set of field lines which define a surface, plus two singular field lines located perpendicular to the surface. On the left-hand side of Figure 7 the

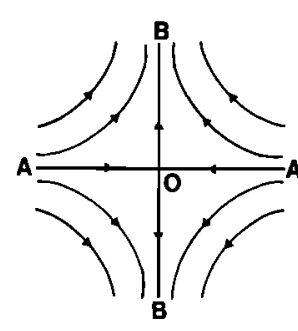

TYPE A
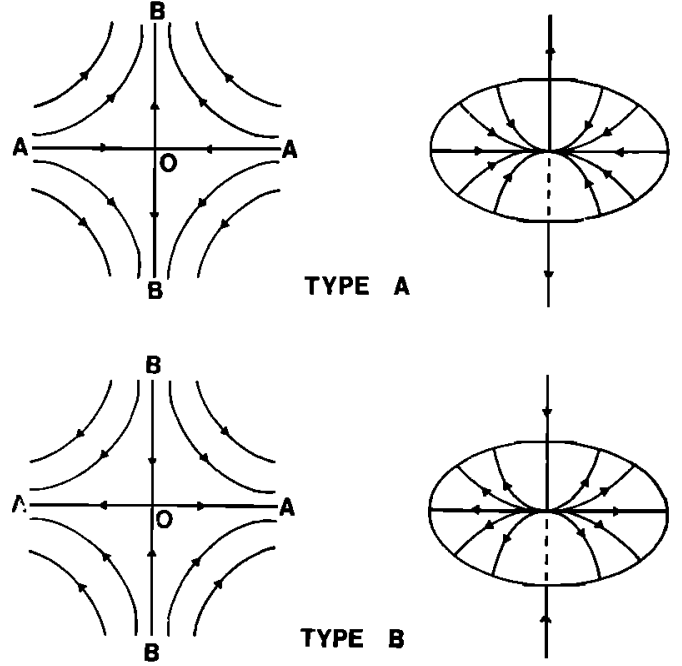

Fig. 7. Field line configurations near neutral points. lines $O A$ lie in the surface as shown on the righthand side while the lines $O B$ are singular field lines.

A second special case of uniform field plus dipole occurs when the imposed field is antiparallel to the dipole moment vector (i.e., a northward interplanetary field). As in the general case two neutral points are formed, one of type A in the terminology of Figure 7, and one of type B. These neutral points lie on the dipole axis, and, as sketched in Figure 8, the surface field lines from the southern type-B neutral point all map along the surface of a sphere into the northern type-A point. They generate the boundary between closed and interplanetary field lines; the open field lines have disappeared (i.e., the magnetosphere is closed) except for the singular field lines from the polar neutral points. We now wish to consider the general case between the two extremes of southward and northward fields which we have discussed. In Figure 9 we show the field lines lying in the plane of the neutral points for this general case. The figure was prepared by assuming that the imposed field is perpendicular to the dipole axis. For the sake of definiteness we might imagine that the imposed field is $B=-\left|B_{I}\right| \hat{y}$ in magnetospheric coordinates so that Figure 9 represents the field lying in the duskdawn meridian plane as seen from the Sun. The neutral points have been chosen to lie at a radial distance from Earth of $15 R_{E}$ corresponding to an interplanetary field strength $B_{I}=13 \gamma$. Because the azimuthal component of the field is nonzero except in this plane, the field lines at other longitudes do not lie in meridians.

In Figure 9 we note that, as in the case of a southward-imposed field, we have three types of field lines, closed, open, and interplanetary, and that the boundary field lines in this plane map from the neutral points. The field-line structure of the lefthand side of Figure 8 is reproduced about these neutral points. We must therefore inquire which of the field lines mapping into the neutral point in Figure 9 belong to the surface and which are the singular lines. A moment's consideration of the field direction out of the plane of the neutral points is sufficient to show that the lines marked $O A$ about each neutral point lie in the surface while $O B$ are the singular lines. The northern neutral point is thus of type A in the terminology of Figure 8 while the southern neutral point is of type $B$. However, even though the lines marked $O A$ lie on a continuous surface of field lines we see that $O A_{1}$ are open while $O A_{2}$ are closed. Therefore, there must exist two field lines 
on the surface, on opposite sides of the neutral point, which mark the boundary between the segment of surface field lines which are open and the segment which is closed. These lines themselves can be neither open nor closed, and hence must map between the neutral points, one around the dayside, the other around the nightside. In the special case of a southward field this special ring-like field line becomes the neutral line encircling the Earth. For our illustrative case of $B_{I}$ perpendicular to the dipole moment vector this field-line ring running between the neutral points is a circle whose radius is equal to the radial distance of the neutral points, and which intersects the $X$ axis at noon and midnight, as shown in Figure 10. With reference to this figure the segment of the surface field lines marked $C_{1} A_{1} C_{2}$ about each neutral point are open lines (i.e., map out to infinity) while the surface segments $C_{1} A_{2} C_{2}$ are closed (i.e., map to the Earth). These segments each define a part of the boundary between the three field-line types, and all these boundaries meet at the ring. For example, the segment $C_{1} A_{2} C_{2}$ of closed field lines from the southern neutral point defines the boundary surface between closed and open field lines in the northern hemisphere, forming the upper half of a doughnut. This surface is cut by the closed singular field line from the northern neutral point $O B_{2}$. As an aid to its visualization we show in Figure 11 a computer-generated $30^{\circ}$ isometric projection of this boundary. The interface between open and closed lines is completed by the surface segment

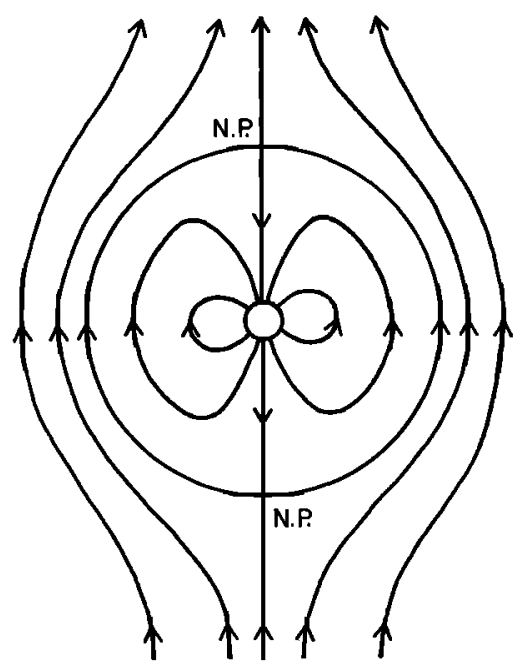

Fig. 8. Field configuration for the special case of a uniform northward magnetic field added to a dipole.

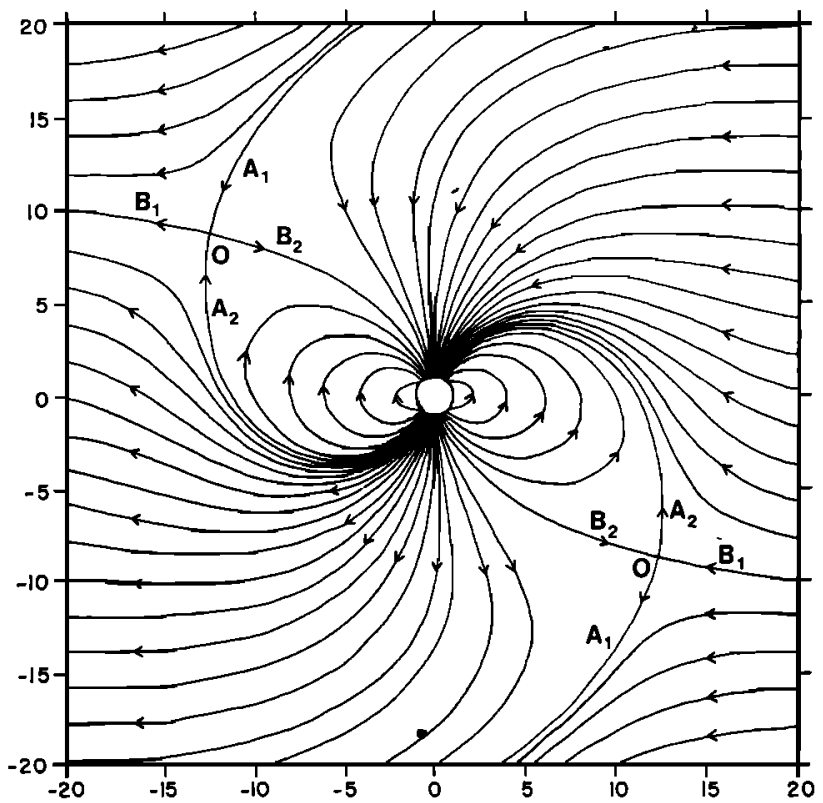

Fig. 9. Field lines in the plane of the neutral points for a uniform field perpendicular to the dipole moment vector.

$C_{1} A_{2} C_{2}$ of the northern neutral point which forms a second half doughnut for the southern hemisphere; this surface is cut by the singular line $O B_{2}$ from the southern neutral point. The remaining surface segments $C_{1} A_{1} C_{2}$ form the surfaces of two

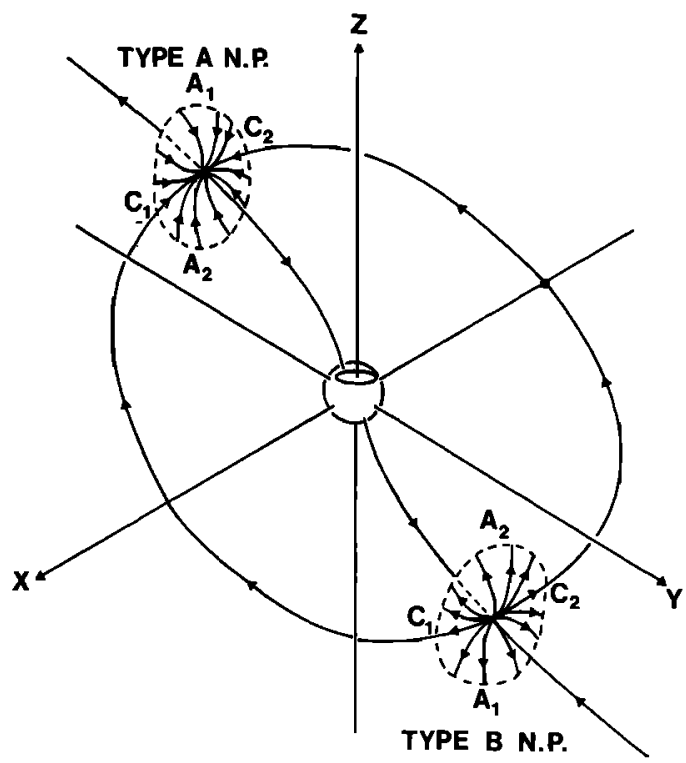

Fig. 10. Three-dimensional arrangement of the neutral points, field-line ring, and surface and singular neutral point field lines. 


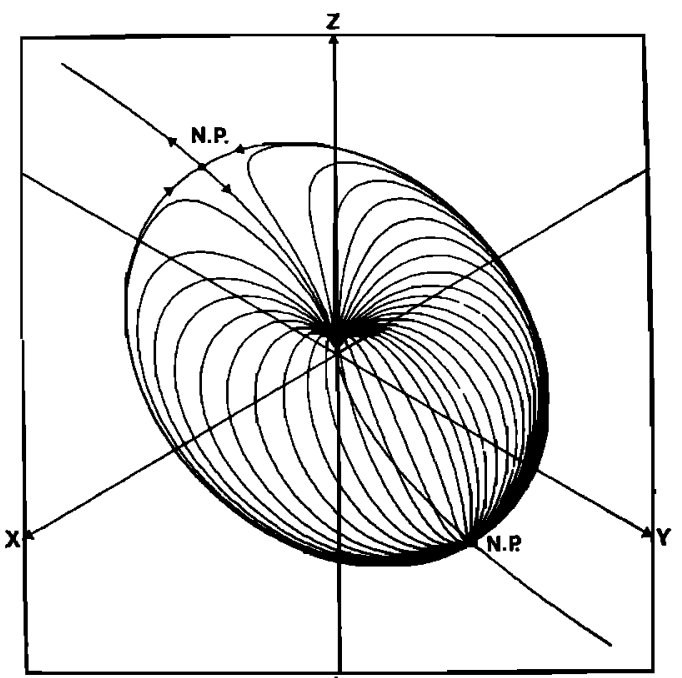

Fig. 11. Isometric $30^{\circ}$ projection of one of the half-doughnut boundaries between open and closed field lines.

cyclinders of open field lines. This segment from the northern neutral point forms the boundary of the north tail lobe and is shown in isometric projection in Figure 12. The surface is cut by the singular neutral point field line $O B_{1}$ from the southern neutral point. (Similarly for the southern tail lobe.)

We now consider the projection of the field lines onto a plane which intersects and is perpendicular to the ring field line. The situation near noon (i.e., equidistant from the neutral points) is sketched in

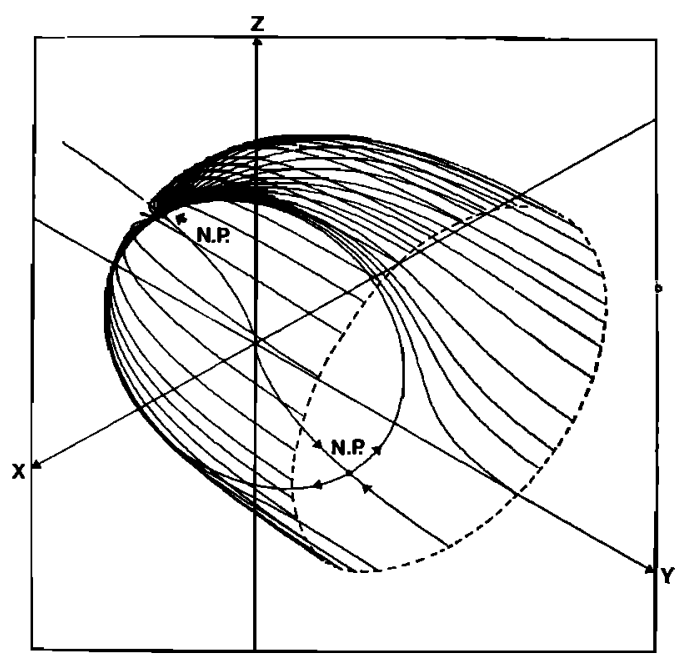

Fig. 12. Isometric $30^{\circ}$ projection of one of the cyclindrical boundaries between open and interplanetary field lines.
Figure 13. An $X$-type configuration is generated, but the center of the $X$ is not a neutral point since there exists a magnetic field component perpendicular to the plane of the projection. The field lines along the separatrices are the field lines in the boundary surfaces between closed, open, and interplanetary lines, and map from either the Earth or the interplanetary medium into one or another of the neutral points. The equivalence of this situation and that discussed above for an infinite $X$-type region should now be clear. In the infinite system the field lines of the separatrices are extended indefinitely along the line running down the center of the $X$; in a finite system they do not extend to infinity but map into neutral points. The field-line ring joining the two neutral points is simply the field line which runs along the center of the $X$ in the infinite system, and thus defines the centers of the day and night diffusion regions. For the finite system, therefore, the diffusion regions lie between and are terminated at neutral points.

\section{THE GENERALIZED RECONNECTION PROCESS IN THE MAGNETOSPHERE}

Having made the above identification of the fieldline ring with the center of the diffusion regions on the dayside and nightside, we can now use the results of the second section to describe qualitatively the reconnection process for the general case. In order to keep the figures as simple and consistent as possible we describe the process in terms of the previous fieldline structures of Figures 9, 10, 11, and 12, and we will not attempt to represent the sweeping back of the reconnected field to produce an antisolar tail, the day-night asymmetry of the magnetosphere, etc. These are the MHD aspects of the problem and will not change the fundamentals of the processes discussed here provided that the topology we have arrived at and its interpretation in terms of the second section are correct. In addition we discuss only dayside reconnection; the nightside process is the same but reversed in time-sequence to that which we discuss here.

In Figure 14 we show the deformation of an equatorial plane interplanetary field line as it is convected up to and into the dayside diffusion region. This line will eventually become connected to a dipole line lying in the noon-midnight plane on the dayside. In (a) the initial interplanetary line $A-A$ is shown. As it enters the diffusion region in (b), it becomes split and extended along the diffusion region 
so that its ends $A$ map through the diffusion region into new partners in the interplanetary convection region. Moving closer to the ring, (c), the extensions along it increase, until in (d) the lines $A$ - $A$ map into the north and south neutral points. At this point they lie in the cylinder boundaries of the open and interplanetary field lines, or equivalently in the open separatrices of the dayside $X$ configuration. The continuation of the lines past the neutral points, as indicated in Figure 14 may be considered to be either the open or the closed singular field lines at the neutral point. At this point a change in the connection of the field lines take place (i.e., from interplanetary to open), that is, reconnection with a dipole field line occurs. We therefore pause to consider the similar progress of the dipole field line to which the original interplanetary line will become connected.

As shown in Figure 15(a) this dipole line lies initially in the noon-midnight plane and is convected toward the ring. As it approaches the ring and moves into the diffusion region it becomes split and extended along it so that the northern part of the line maps into a new partner on the dusk side of noon while the southern part maps into a new partner on the dawn side of noon, (b). This description is valid for $B_{y_{I}}<0$, the change of connection with respect to noon is reversed for positive $B_{y}$. Moving closer to the ring the extension increases, (c), until the lines map to the neutral points (d). They then lie in the boundary of the closed field-line doughnut, or equivalently in the closed separatrices of the dayside $X$ type configuration. As in Figure 14(d) the continuation of these lines out from their neutral points may be considered to be either the closed or open singular line from that point.

In Figure 16(a) we show the northern part of the original dipole line, now mapping to the southern neutral point as in Figure 15(d), and that part of the original interplanetary line which maps to the

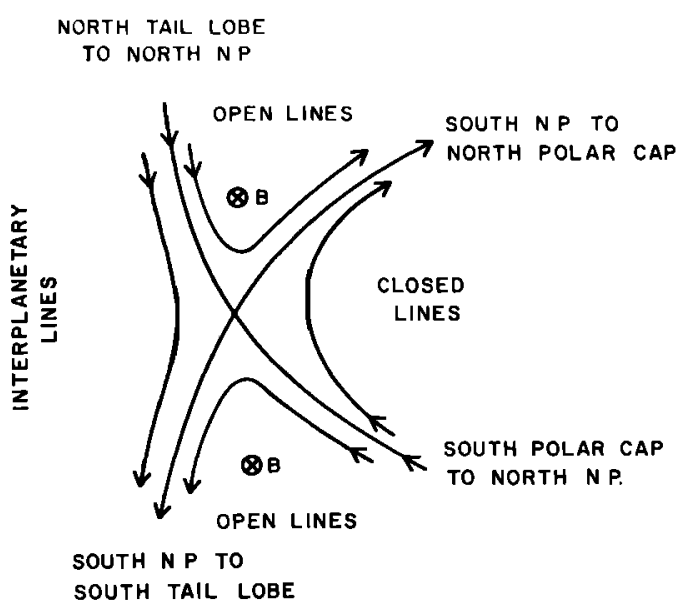

Fig. 13. Projection of the field lines near the ring onto a plane perpendicular to the ring at noon.

northern neutral point as in Figure 14(d). These two will become connected when they are outside the diffusion region. Following through the histories as initiated for these two parts in Figures 14 and 15 the field lines pass through the separatrices so that the interplanetary line now becomes open and maps to the polar cap near dawn, while the dipole line also becomes open and maps into the interplanetary medium near the open singular line from the southern neutral point, Figure 16(b). As these lines move up and away from the ring the mapping of the interplanetary lines into the polar cap moves toward noon, while the original dipole line maps into the interplanetary medium closer to the original interplanetary line, since their extensions along the ring are being reduced, Figure 16(c). Finally, out of the diffusion region, the two lines are joined and flow as a single open field line into the north tail lobe, Figure 16(d).
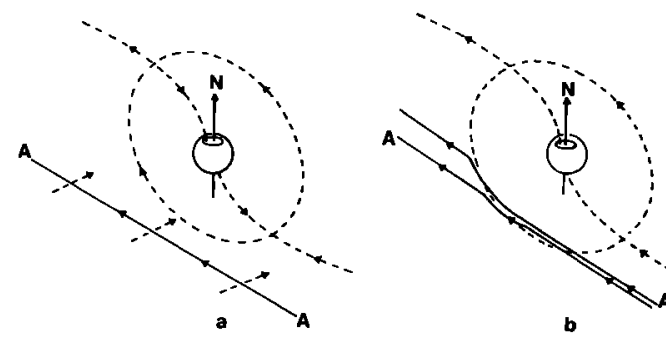
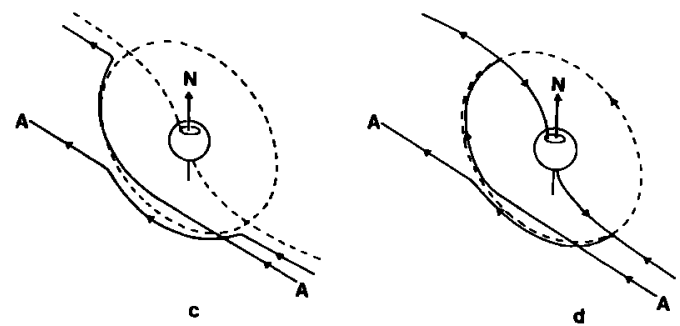

Fig. 14. Deformation of an interplanetary field line as it convects into the dayside diffusion region. 


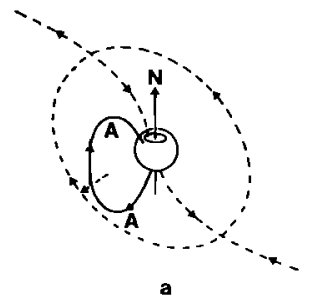

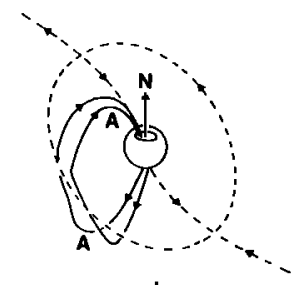

b
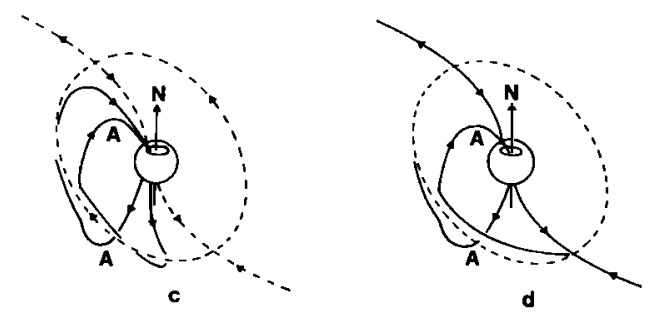

Fig. 15. Deformation of a dipole field line as it is convected into the dayside diffusion region.

\section{DISCUSSION}

In the preceding sections we have shown how the qualitative description of the reconnection process must be modified when the reconnecting fields are not exactly antiparallel. A splitting of the incoming field lines and a continuous exchange of partners along the $X$ line takes place when a field line maps into the diffusion region. Such a picture of the process is just that required to be consistent with the expected topology of the magnetosphere for nonantiparallel fields. We conclude this paper with a brief discussion of the electric fields.

Outside the diffusion region the equation

$$
\mathbf{E}=-\mathbf{v} \times \mathbf{B} / c
$$

is expected to be valid, so that the field lines are equipotentials. In the diffusion region finite conductivity effects are dominant, the above frozen-in equation no longer applies, and field lines are not necessarily equipotentials. In particular, it is obvious that the field line which runs down the center of the $X$ for the nonantiparallel field case is not an equipotential. In the finite geometry this implies that there exists a potential drop between the neutral points, corresponding to the potential drop across the polar caps. The field lines which lie in the various boundary surfaces all map to one or another of the two neutral points. However, in the convection regions the field lines on one of these boundary surfaces are not the same potential, but the potential varies between that of the two neutral points. For example, if we consider one of the tail lobes as in Figure 12, the singular field line which cuts the surface from the southern neutral point is expected to be at southern neutral-point potential. As we move around the cylinder surface we expect the potential to vary monotonically until northern neutral-point potential is reached on the field line at the top of the cylinder. However, all these field lines map to the northern neutral point. A similar argument applies to the two closed field-line boundary surfaces. The situation is not inconsistent however, since all these field lines pass through the dayside or nightside diffusion regions in their mapping to the neutral point. The potential drop in the diffusion region which occurs along one of these field lines simply corresponds to the difference between its potential in whichever convection region it lies and the potential of the neutral point to which it maps.

The magnetospheric and ionospheric convection corresponding to these models will be the same on a large scale as that previously discussed for a purely southward interplanetary field [Dungey, 1961], that is, a dawn-to-dusk electric field across the polar cap corresponding to the motion of open lines over the cap into the tail, with the ionospheric return flow at lower latitudes, corresponding to the flow of tailreconnected closed field lines towards the dayside in the equatorial plane.
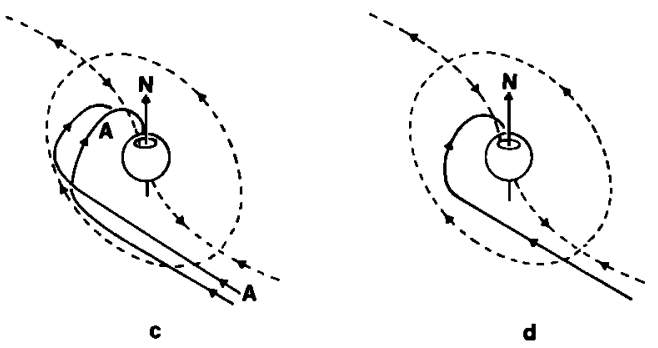

Fig. 16. The reconnection process completed. 
Acknowledgments. This work was performed while the author was a Visiting Fellow at the Cooperative Institute for Research in Environmental Sciences, Boulder, Colorado. The author would like to thank the faculty and staff of CIRES for their help and hospitality during the year's tenure of the Fellowship.

\section{REFERENCES}

Alfvén, H. (1968), Some properties of magnetospheric neutral surfaces, J. Geophys. Res., 73, 4379-4381.

Cowley, S. W. H. (1973), A self-consistent model of a simple magnetic neutral sheet system surrounded by a cold, collisionless plasma, Cosmic Electrodyn., 3, 448501.

Dungey, J. W. (1961), Interplanetary magnetic field and the auroral zones, Phys. Rev. Letters, 6, 47-48.

Dungey, J. W. (1963), The structure of the exosphere or adventures in velocity space, in Geophysics, The Earth's Environment, edited by C. DeWitt, J. Hieblot, and L. LeBeau, pp. 503-550, Gordon and Breach, New York.
Eastwood, J. W. (1972), Consistency of fields and particle motion in the 'Speiser' model of the current sheet, Planet. Space Sci., 20, 1555-1568.

Gonzales, W., and F. S. Mozer (1973), High latitude electric fields and the three-dimensional interaction between the interplanetary and terrestrial magnetic fields, Ser. 13, issue 103, 38 pp., Space Science Laboratory, University of California, Berkeley, Calif.

Harris, E. G. (1962), On a plasma sheath separating regions of oppositely directed magnetic field, $\mathrm{Nuovo} \mathrm{Ci}$ mento, 23, 115-121.

Sonnerup, B. U. O. (1970), Magnetic field re-connexion in a highly conducting incompressible fluid, J. Plasma Phys., 4, part 1, 161-174.

Stern, D. P. (1972), A study of the electric field in an open magnetospheric model, $X-641-72-463,25$ pp., Goddard Space Flight Center, Greenbelt, Md.

Yeh, T., and W. I. Axford (1970), On the re-connexion of magnetic field lines in conducting fluids, J. Plasma Phys., 4, part 2, 207-229. 